\title{
Properties of Dust in Giant Elliptical Galaxies: The rôle of the Environment
}

\author{
Paul Goudfrooij* \\ Space Telescope Science Institute, 3700 San Martin Drive, Baltimore, \\ MD 21218, U.S.A.
}

\begin{abstract}
.
The properties of dust in giant elliptical galaxies are reviewed, with particular emphasis on the influence of the environment.

After normalizing by the optical luminosities, a strong anticorrelation between the masses of dust and hot gas in X-ray bright ellipticals is found. Furthermore, large-scale, regularly-shaped dust lanes (which are symmetric with respect to the galaxy nucleus) are only found to be present in ellipticals with the lowest ratios of the mass of hot gas to the blue luminosity $\left(M_{\text {hot }} / L_{B} \lesssim 0.04\right.$ in solar units). This can be explained by the short time scale for the destruction of dust grains within the hot, $\mathrm{X}$ ray-emitting gas compared to the formation timescale of such dust lanes in early-type galaxies.

Dust within ellipticals in "loose" environments (i.e. in the field or in loose groups) is typically characterized by small values of $R_{V} \equiv$ $A_{V} / E_{B-V}$ (i.e. small characteristic grain sizes), and distributed in dust lanes with a smooth, relaxed morphology. On the other hand, dust in ellipticals that are in or near the center of dense groups or clusters is typically much more irregularly distributed, and characterized by $R_{V}$ values that are close to the Galactic one.

I predict that ellipticals containing "relaxed" dust lanes with typical dust masses of $10^{6}-10^{7} M_{\odot}$ do not contain hot, X-ray-emitting gas.
\end{abstract}

\section{Introduction: The Multi-Phase ISM of Elliptical Galaxies}

It has become evident in recent years that elliptical galaxies are far from being the simple, (violently) relaxed, isothermal, purely stellar systems anticipated by the traditional picture developed by Hubble. Recent deep surveys across the electromagnetic spectrum have shown that ellipticals contain a complex, multiphase interstellar medium (ISM). In fact, all ISM components known to exist in spiral galaxies are now accessible in elliptical galaxies as well, although in rather different proportions. The main difference is that the dominant (in mass) gaseous component in spirals is "cool", in the form of neutral gas $\left(\mathrm{H} \mathrm{I}_{2} \mathrm{H}_{2}\right)$, whereas in ellipticals it is "hot" $\left(\sim 10^{7} \mathrm{~K}\right)$, radiating at $\mathrm{X}$-ray wavelengths. The typical mass of this hot gas has been found to be of order $10^{9}-10^{11} M_{\odot}$ (for

\footnotetext{
${ }^{1}$ Affiliated to the Astrophysics Division, Space Science Department, European Space Agency
} 
$\mathrm{H}_{0}=50 \mathrm{~km} \mathrm{~s}^{-1} \mathrm{Mpc}^{-1}$ ), which is similar to the expected amount from stellar mass loss accumulated over the lifetime of luminous ellipticals (e.g. Forman et al. 1985; Loewenstein 1998). Hence, it is now commonly believed that the hot gas is indeed originated - and constantly replenished- by mass loss of stars within the ellipticals which is thermalized in the gravitational field of the galaxies (i.e. with temperature $T_{\sigma}=\mu m_{p} \sigma_{*}^{2} / k$, where $\mu$ is the mean atomic weight, $m_{p}$ is the proton mass, and $\sigma_{*}$ is the stellar velocity dispersion).

It should be noted that evidence for the existence of this hot component of the ISM is currently only substantial for the most luminous ellipticals $\left(L_{B} \gtrsim\right.$ $510^{10} L_{\odot}$, see e.g. Kim et al. 1992), as well as for ellipticals that are in the centers of groups (Mulchaey \& Zabludoff 1998). It thus seems that only those ellipticals that are "privileged" to reside within a deep enough potential well are able to retain the material lost by stars and suppress the supernova-driven wind proposed by Mathews \& Baker (1971). In this scenario, smaller ellipticals with too shallow potential wells may not see the bulk of their internally produced ISM ever again, donating it to the intracluster or intragroup medium.

Along with the "hot" ISM, the cooler ISM components exist in ellipticals as well: "warm" ionized gas (Goudfrooij 1998; Macchetto et al. 1996), dust (Knapp et al. 1989; Goudfrooij \& de Jong 1995), and "cold" CO and H I (Lees et al. 1991; Wiklind et al. 1995; Oosterloo et al. 1998). However, the measured amounts of dust, cold gas and warm gas are typically small, and do not correlate with the stellar luminosity of ellipticals (as opposed to the case among spirals, cf. Lees et al. 1991). While it is conceivable that some part of the observed dust and gas originates from stellar mass loss, this observational result indicates that most of it has an external origin (e.g. accreted during an interaction with a smaller, gas-rich galaxy). See also Forbes (1991) and Goudfrooij \& de Jong (1995).

Typical properties of the ISM in ellipticals are listed below in Table 1 (for comprehensive reviews, see e.g. Goudfrooij 1997, 1998; Knapp 1998).

Table 1. The Multi-Phase ISM of Elliptical Galaxies

\begin{tabular}{lrrcc}
\hline \hline ISM Phase & \multicolumn{1}{c}{ Temp. } & \multicolumn{1}{c}{$f^{a}$} & \multicolumn{1}{c}{ Mass } & Probable Origin \\
\hline Molecular & $\lesssim 10 \mathrm{~K}$ & $\sim 8 \%$ & $\Im 10^{7} M_{\odot}$ & External \\
Dust & $\sim 15-100 \mathrm{~K}$ & $245 \%$ & $10^{4}-10^{7} M_{\odot}$ & External \\
H I & $\sim 100 \mathrm{~K}$ & $\sim 10 \%$ & $10^{8}-10^{9} M_{\odot}$ & External \\
H ${ }_{\text {II }}$ & $\sim 10^{4} \mathrm{~K}$ & $\sim 70 \%$ & $10^{3}-10^{5} M_{\odot}$ & External \\
Hot & $\sim 10^{7} \mathrm{~K}$ & $\sim 70 \%$ & $10^{9}-10^{11} M_{\odot}$ & Internal \\
\hline
\end{tabular}

${ }^{a} f \cong$ Detection fraction

\section{Properties of Dust within Ellipticals in Different Environments}

Dust features have been found to exist in about half of all nearby ellipticals. Dust lanes or patches are known to exist in small ellipticals as well as in giant ellipticals, whereas the latter often reside in environments of high galaxy density and/or massive halos of hot gas. This leads me to the main issue I wish to discuss in this contribution: Do the properties of dust (e.g. grain size, dust content, morphology) vary among ellipticals due to differences in the environment? 
Most detailed studies of the properties of dust in ellipticals have been undertaken by means of optical imaging, thanks to its intrinsically high spatial resolution and the inherently smooth optical light distributions of ellipticals which eases the comparison of extinguished areas of the galaxies with the appropriate unextinguished areas (e.g., Goudfrooij et al. 1994b). As the extinction in the optical regime $(\lambda \sim 4000-7000 \AA)$ is caused by grains with a typical size of $\sim 0.1 \mu \mathrm{m}$, it is worth taking a look at the typical grain destruction time scales $\left(\tau_{\mathrm{d}}\right)$ of different mechanisms that might cause any size change for $0.1 \mu \mathrm{m}$-sized dust grains within ellipticals. These mechanisms are summarized below:

1. Grain-grain collisions in low-velocity $\left(\$ 50 \mathrm{~km} \mathrm{~s}^{-1}\right)$ shocks: $\tau_{\mathrm{d}} \sim 1 \times 10^{9} \mathrm{yr}$ (e.g. Jones et al. 1996). This velocity was selected since it is the typical maximum velocity dispersion in nebular emission lines within ellipticals (e.g. Goudfrooij 1998).

2. Sputtering in supernova-driven blast waves in a two-phase medium (cold dense clouds embedded within a coronal intercloud medium of low density): $\tau_{\mathrm{d}} \sim$ $3 \times 10^{9}\left(L / 10^{10} L_{\odot}\right)^{-1}$ yr (Draine \& Salpeter [1979], using the current supernova explosion rate within ellipticals from Turatto et al. [1996] and $\mathrm{H}_{0}=50 \mathrm{~km} \mathrm{~s}^{-1} \mathrm{Mpc}^{-1}$ ).

3. Sputtering by thermal ions: (a) "Warm" $10^{4} \mathrm{~K}$ gas: $\tau_{\mathrm{d}} \sim 10^{10} \mathrm{yr}$ (Barlow $1978)$; (b) "Hot", $\mathrm{T} \sim 10^{7} \mathrm{~K}$ gas: $\tau_{\mathrm{d}} \simeq 2 \times 10^{5}\left(n_{p} / \mathrm{cm}^{-3}\right)^{-1}(a / 0.1 \mu \mathrm{m})$ yr (e.g. Tielens et al. 1994).

Note that the latter destruction time scale is typically only $\lesssim 10^{7}$ yr for 0.1 $\mu \mathrm{m}$ grains (and proportionally shorter for smaller grains) within the central few $\mathrm{kpc}$ of X-ray bright ellipticals where the typical proton density $n_{p} \sim 0.03-0.1$ $\mathrm{cm}^{-3}$ (see, e.g. Trinchieri et al. 1997). Sputtering by hot ions (protons, He nuclei) is therefore the dominant destruction agent by far for dust in ellipticals embedded in hot gas. One would therefore expect the dust content of ellipticals to decrease with increasing $\mathrm{X}$-ray flux, while the dust grain size distribution in $\mathrm{X}$-ray bright ellipticals is expected to be depleted in small grains. On the other hand, if sputtering by hot ions is not the dominant grain destruction agent (e.g. in ellipticals not embedded in hot gas), destruction mechanism (1) above may be dominant, which preferentially destroys large grains.

To address the correctness of these suggested relations, I selected from the catalog of galaxies observed by EINSTEIN (Fabbiano et al. 1992) the ellipticals with $L_{X} / L_{B} \geq 2 \times 10^{30} \mathrm{erg} \mathrm{s}^{-1} \mathrm{~L}_{\odot}^{-1}$, for which Kim et al. (1992) showed that the $\mathrm{X}$-ray flux is dominated by emission from hot gas. For those ellipticals, dust masses were derived from the IRAS 60 and $100 \mu$ m fluxes (Knapp et al. 1989) as described in Goudfrooij \& de Jong (1995). Masses of hot gas were derived from the EINSTEIN fluxes according to eq. (9) of Canizares et al. (1987). Keep in mind that these gas masses are uncertain by a factor of $2-4$ due to the uncertainties connected with deriving density profiles from EINSTEIN data. Fig. 1 depicts the relation of the masses of dust and hot gas for X-ray bright ellipticals. The masses have been divided by the optical luminosities of the galaxies to remove the (distance) ${ }^{2}$ factor. And indeed, a strong anticorrelation between the masses of dust and hot gas is seen, as expected (cf. above). 


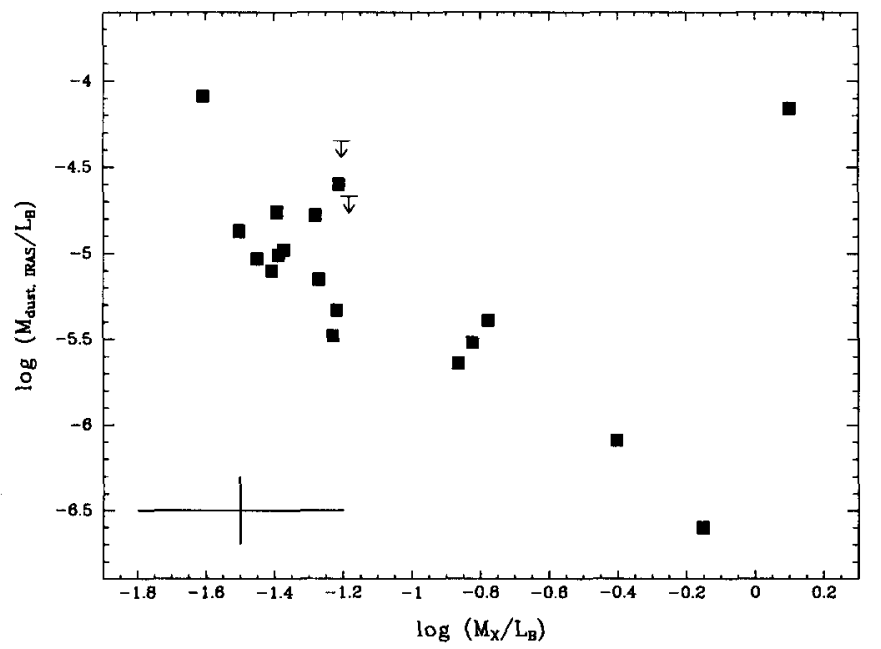

Figure 1. The relation of the ratio of dust mass to blue luminosity with the ratio of the mass of hot, X-ray-emitting gas to the blue luminosity for elliptical galaxies with $L_{X} / L_{B} \geq 2 \times 10^{30} \mathrm{erg} \mathrm{s}^{-1} \mathrm{~L}_{\odot}^{-1}$. Typical errorbars are shown in the bottom left corner.

The single data point that deviates strongly from the general trend in Fig. 1 represents NGC 4696 , the dominant elliptical in the Centaurus cluster. This galaxy is however expected to be a special case in view of the presence of a filamentary, obviously "young" dust structure in its central region. de Jong et al. (1990) and Sparks et al. (1989) have suggested that the dust in NGC 4696 was captured during a recent $\left(\sim 10^{8}\right.$ yr ago) tidal interaction with a smaller, gas-rich galaxy, while the dust can be replenished during $\sim 10^{8}$ yr by evaporation of cool clouds (captured during the interaction) by hot electrons within the hot gas.

What about the dust grain size distribution? Goudfrooij et al. (1994b) measured extinction curves for dust in 10 ellipticals that display obvious dust extinction features superposed on an otherwise smooth distribution of light (following a de Vaucouleurs law). Interestingly, they found $R_{V} \equiv A_{V} / E_{B-V}$ to be very small for a number of giant ellipticals with large-scale dust lanes having relaxed morphologies $\left(R_{V} \sim 2.1-2.7\right.$, whereas $R_{V}=3.1$ in the diffuse ISM in our Galaxy), which means that the characteristic grain size for the grains causing optical extinction is significantly smaller in those galaxies than in ours. Conversely, the optical extinction curve in X-ray bright ellipticals turns out to be more "normal", with $R_{V}$ values that are consistent with the Galactic value (Sparks et al. 1989; Goudfrooij \& Trinchieri 1998). In Fig. 2, I show a comparison of the distribution of dust extinction (through a $B-I$ image) with the extinction curve for two representative cases: IC 4320 , an isolated elliptical with a relaxed, large-scale dust lane along its minor axis, and NGC 4696, the central, X-ray bright galaxy of the Centaurus cluster (see above).

Unfortunately, none of the giant ellipticals with large-scale dust lanes for which Goudfrooij et al. (1994b) derived $R_{V}$ values that are significantly smaller than the Galactic value has been observed in X-rays yet (except for the ROSAT 
all-sky survey, which was however too shallow to detect any significant emission by hot gas from these ellipticals). Moreover, none of them show any other sizeable galaxies within a radius of several $10^{2} \mathrm{kpc}$ around them on Digital Sky Survey images, and they are not in any group catalog (e.g. Garcia 1993). They may well be field ellipticals which stripped dust (and gas) from small neighboring galaxies, after which no significant replenishment of dust has occurred in the lanes. In the absence of a massive hot gas halo, the characteristic dust grain size will then slowly decrease through grain-grain collisions, as observed.

Recent models of galaxy interactions involving an elliptical-spiral pair have shown that the settling time scale for gas disks over radial extent of $\sim 10 \mathrm{kpc}$ (which is typical for the large-scale dust lanes in these isolated ellipticals) is at least a few tens of crossing times (i.e. $\sim 3 \times 10^{9} \mathrm{yr}$; e.g. Steiman-Cameron \& Durisen 1990). Note that the grain destruction time scale in hot gas $\left(\tau_{\mathrm{d}} \lesssim 10^{7}\right.$ yr, see above) is $100-300$ times shorter than this. Hence, one would predict that ellipticals with such large-scale dust lanes do not host massive hot gas haloes. If this prediction proves true, the $L_{X} / L_{B}$ ratio for these luminous $\left(L_{B} \gtrsim 10^{11} L_{\odot}\right)$ ellipticals would be significantly lower than any observed so far by EINSTEIN or ROSAT. This would provide strong evidence for a scenario in which the potential wells of single galaxies are not deep enough to retain the stellar mass loss. Only ellipticals located in the centers of (sub-)clusters or rich groups would be able to stifle the galactic winds. One will be able to resolve this question using the highly sensitive EPIC camera aboard the X-ray satellite $X M M$.

Acknowledgments. It is a pleasure to thank the organizing committee of this conference for an exciting and fruitful meeting.

\section{References}

Barlow, M., 1978, MNRAS 183, 367

Canizares, C. R., Fabbiano, G., Trinchieri, G., 1987, ApJ 312, 503

de Jong, T., Nørgaard-Nielsen, H. U., Hansen, L., Jørgensen, H. E., 1990, A\&A 232,317

Draine, B. T., Salpeter, E., 1979, ApJ 231, 77

Forbes, D. A., 1991, MNRAS 249, 779

Fabbiano, G., Kim, D.-W., Trinchieri, G., 1992,ApJS 80, 531

Forman, W. R., Jones, C., Tucker, W., 1985, ApJ 293, 102

Garcia, A. M., 1993, A\&AS 100, 47

Goudfrooij, P., 1997, in: The Nature of Elliptical Galaxies, eds. M. Arnaboldi, G. S. da Costa, \& P. Saha, ASP, San Francisco, p. 338

Goudfrooij, P., 1998, in: Star Formation in Early-type Galaxies, eds. J. Cepa \& P. Carral, ASP, San Francisco, p. 55

Goudfrooij, P., Hansen, L., Jørgensen, H. E., Nørgaard-Nielsen, H. U., 1994a, A\&AS 105, 341

Goudfrooij, P., de Jong, T., Hansen, L., Nørgaard-Nielsen, H. U., 1994b, MNRAS 271, 833

Goudfrooij, P., de Jong, T., 1995, A\&A 298, 784 
Goudfrooij, P., Trinchieri, G., 1998, A\&A 330, 123

Jones, A.P., Tielens, A. G. G. M., Hollenbach, D. J., 1996, ApJ 469, 740

Kim, D.-W., Fabbiano, G., Trinchieri, G., 1992, ApJ 393, 134

Knapp, G. R., 1998, in: Star Formation in Early-type Galaxies, eds. J. Cepa \& P. Carral, ASP, San Francisco, p. 119

Knapp, G. R., Guhathakurta, P., Kim, D.-W., Jura, M., 1989, ApJS 70, 329

Lees, J. F., Knapp, G R., Rupen, M. P., Phillips, T. G., 1991, ApJ 379, 177

Loewenstein, M., 1998, in: Galactic and Cluster Cooling Flows, ed. N. Soker, ASP, San Francisco, p. 100

Macchetto, F., Pastoriza, M., Caon, N., et al. 1996, A\&AS 120, 463

Mathews, W. G., Baker, J. C., 1971, ApJ 170, 241

Mulchaey, J. S., Zabludoff, A. I., 1998, ApJ 496, 73

Oosterloo, T. A., Morganti, R., Sadler, E. M., 1998, in: Star Formation in Earlytype Galaxies, eds. J. Cepa \& P. Carral, ASP, San Francisco, p. 72

Roberts, M.S., Hogg, D.E., Bregman, J.E., Forman, W. R., Jones, C., 1991, ApJS 75, 751

Sparks, W. B., Macchetto, F., Golombek, D., 1989, ApJ 345, 513

Steiman-Cameron, T. Y., Durisen, R. H., 1990, ApJ 357, 62

Tielens, A.G. G. M., McKee, C.F., Seab, C.G., Hollenbach, D. J., 1994, ApJ 431,321

Trinchieri, G., Noris, L., di Serego Alighieri, S., 1997, A\&A 326, 56528

Wiklind, T., Combes, F., Henkel, C., 1995, A\&A 297, 643 

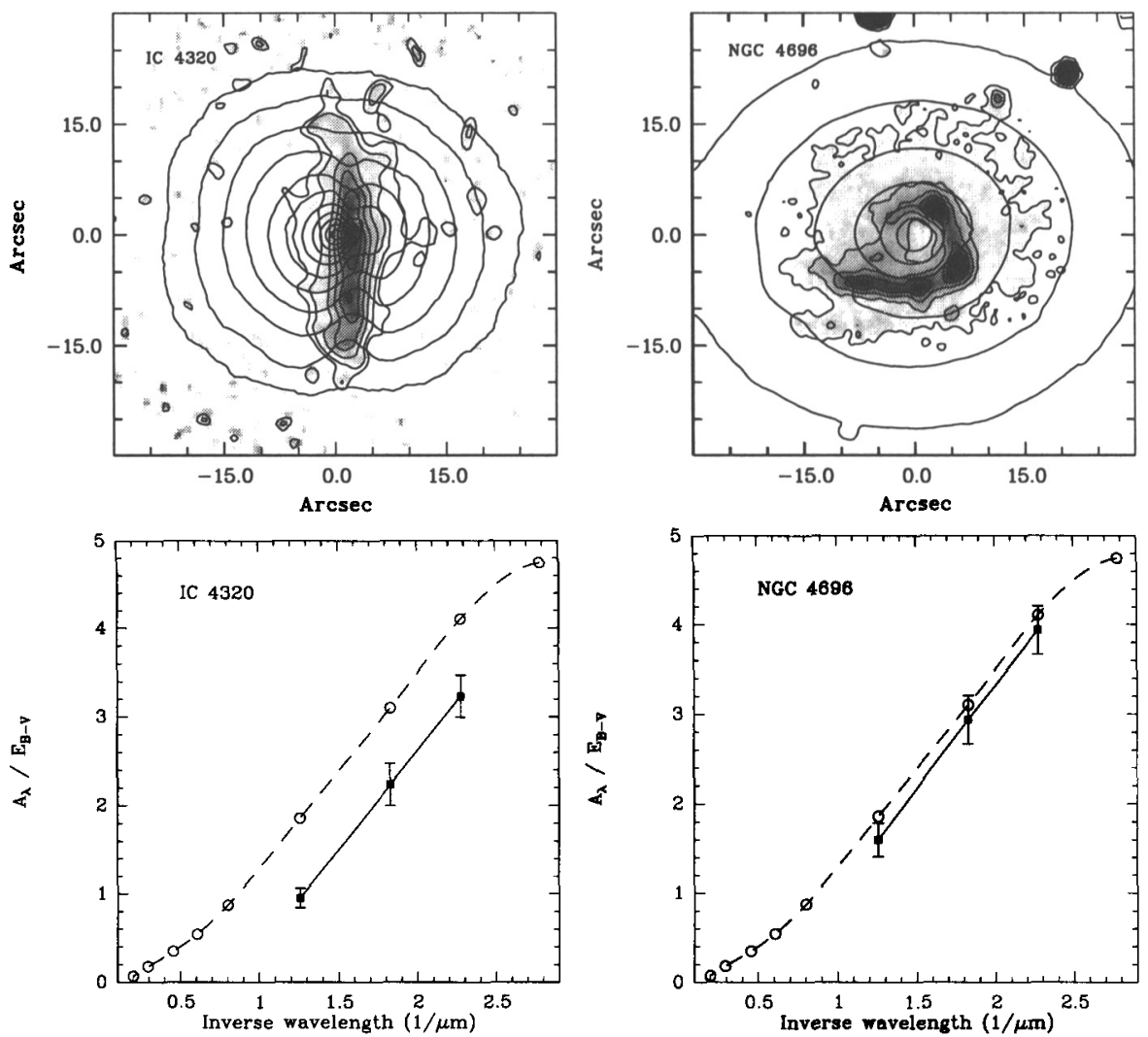

Figure 2. An illustration of the difference in properties of dust in giant elliptical galaxies in different environments (see text for details). The top panels show $B-I$ color-index images (grey scales) with isophotal contours of the $B$-band image superposed (thick solid lines). The bottom panels show optical $(B, V, I)$ extinction curves of the galaxies (filled squares, solid lines) superposed on the Galactic extinction curve (open circles, dashed lines) for comparison. Left: IC 4320, an isolated elliptical. Note the relaxed dust lane morphology and the small value of $R_{V}$, indicating small grains. Right: NGC 4696. Note the filamentary dust morphology and the "normal" extinction curve. Images taken from Goudfrooij et al. (1994a, 1994b). 